Intermediale Relationen 

Ludwig Jäger

\title{
Intermedialität - Intramedialität - Transkriptivität Überlegungen zu einigen Prinzipien der kulturellen Semiosis
}

\begin{abstract}
In den unterschiedlichsten disziplinären Kontexten der Kultur- und Medienwissenschaften findet in den beiden letzten Jahrzehnten der Befund große theoretische Aufmerksamkeit, dass Medien intermedial aufeinander Bezug nehmen (Bolter/ Grusin 2001) und dass sie sich intramedial in rekursiven Schleifen auf sich selbst beziehen. Die kulturelle Semiosis wird - folgt man diesen Studien - auf einem Feld generiert, konserviert, gestört und fortgeschrieben, auf dem sich Kommunikation als semiologisches Spiel interagierender und miteinander verwobener Medien, als ein Ensemble von Praktiken „kultureller Rekonzeptualisierung“ (Manovich 2001) entfaltet. Der Beitrag vertritt die These, dass sich hinter der Vielfalt dieser kommunikativen Prozeduren in den Sprach-, Schrift- und Bildmedien, so unterschiedlich sie auch in medialer und ästhetischer Hinsicht sind, ein grundlegendes Verfahren der kulturellen Semiosis verbirgt, das sich als ein Verfahren transkriptiver Bezugnahme beschreiben lässt. Die Annahme, dass Transkription eine grundlegende Operation der kulturellen Semiosis, d.h. der Prozeduren kultureller (intraund intermedialer) Rekonzeptualisierung, darstellt, soll anhand einiger epistemologischer und zeichentheoretischer Prinzipien erläutert und theoretisch verständlich gemacht werden.
\end{abstract}

\section{Vorbemerkung}

Es ist genau zehn Jahre her, dass die Jahrestagung des Instituts für Deutsche Sprache mit dem Thema „Sprache und neue Medien“ den disziplinären Diskurs der germanistischen Sprachwissenschaft nachdrücklich auf seine theoretische Positionierung zu jenem grundlegenden Wandel der Kommunikationsverhältnisse hin befragte, der mit der globalen Evolution der sogenannten ,neuen Medien' verbunden war. Im Vorwort des Tagungsbandes dieser Tagung formulierte Werner Kallmeyer: „Der Ausdruck ,neue Medien im Titel ,Sprache und neue Medien' zielt auf elektronische Medien wie Computer und Internet, die sich in kurzer Zeit eine zentrale Stellung in der gesellschaftlichen Kommunikationsstruktur erobert haben“" (Kallmeyer 2000a, S. VII). Ohne Zweifel stellte diese Konferenz einen wichtigen Impuls für eine umfassendere Etablierung des Medienthemas in der germanistischen Sprachwissenschaft dar - bis hin zur Reflexion der Nutzung neuer Medien als methodischer Forschungsinstrumente. Freilich blieb der thematische Fokus der Jahrestagung von 1999 noch vornehmlich auf den Funktionswandel von Sprache in den neuen Medien gerichtet, d.h. - wie es 
ebenfalls bei Kallmeyer heißt - auf den „Wandel von Sprache und Kommunikationsformen unter dem Einfluss der neuen Medien“ (Kallmeyer 2000b, S. 293). So verdienstvoll dieses vor allem in der letzten Dekade prosperierende linguistische Forschungsparadigma nun ohne Zweifel nach wie vor ist, so adressiert es die Sprache doch eher als Patienten medialer Akteure, d.h. eher als Gegenstandsfeld medialer Wirkungen, denn als symbolisches System, das selbst Medium ist. Eben diese Kehrseite des Themenfeldes „Sprache und Medien“ nahm nun im letzten Jahrzehnt insbesondere der medientheoretische Diskurs der Kulturwissenschaften in den Blick, der nachdrücklich dazu beigetragen hat, die Medialität des Mediums Sprache selbst stärker in den Fokus der Aufmerksamkeit zu rücken (Jäger/Linz (Hg.) 2004; Krämer 2004), d.h. die Klärung einer Frage voranzubringen, die ich mir erlaubt habe, hier vor zehn Jahren zu stellen, nämlich „ob wir nicht $[\ldots]$ - bei aller Notwendigkeit, die immer komplexer werdenden Verhältnisse von Sprache und (neuen) Medien zu bestimmen - immer zugleich auch die Frage nach der Medialität des anthropologischen Rahmenmediums Sprache stellen müssen, weil sich vielleicht erst von hier aus Aufklärung darüber gewinnen läßt, was die Medialität von Medien ausmacht" (Jäger 2000, S. 10). Auch wenn über die - in dieser Frage versteckte, vielleicht anmaßend anmutende - kulturelle und theoretische Privilegierung der Sprache vor anderen Medien sicher kein konfliktfreies Einvernehmen in den Kulturwissenschaften herrscht - insbesondere nicht etwa aus der Perspektive des rezenten bildtheoretischen Diskurses (Mitchell 1995/2008; Boehm (Hg.) 1995; Boehm/Mersmann/Spies (Hg.) 2008) -, zeigen die intensiven Debatten über Sprache und Schrift (Stetter 1997; Grube/Kogge/Krämer (Hg.) 2005), über Sprache und Stimme (Waldenfels 1999; Epping-Jäger/Linz (Hg.) 2003; Kolesch/Krämer (Hg.) 2006), über Sprache und Gestik (Kendon/ Müller 2007 ff.), über das Verhältnis von Laut- und Gebärdensprachen (Fehrmann/Jäger 2004) sowie schließlich nicht zuletzt über die Beziehungen zwischen linguistic und pictorial turn (Mitchell 1995/2008), dass die Sprache selbst als ein multimodales (Fricke 2008) und zudem in verschiedenen medialen Formaten auftretendes Medium (Jäger 2001), wenn man so will, als ein Intermedium, sowohl in den Kulturwissenschaften allgemein als auch in der Sprachwissenschaft - wie insbesondere auch das Programm dieser Tagung zeigt - wieder erhöhte theoretische Aufmerksamkeit genießt. In der Sprachwissenschaft zeigt sich zudem in der Hinwendung zur Medialitätsdimension der Sprache zugleich zumindest partiell eine Abkehr vom medialitätsfeindlichen Paradigma des linguistischen Kognitivismus und das heißt von dem, was ich die „Medialitätsvergessenheit der Sprachtheorie“ (Jäger 2000, S. 26 ff.) genannt habe.

Der sich deutlich belebende Diskurs über Medialität im Allgemeinen und Sprachmedialität im Besonderen, von dem sich offenbar auch das Pro- 
gramm dieser Tagung anregen ließ, hat sich im Horizont eines breiteren medialitätstheoretischen Forschungsfeldes in den Kulturwissenschaften entfaltet, das inzwischen den gemeinsamen theoretischen Rahmen für eine Reihe von geistes- und kulturwissenschaftlichen Einzeldisziplinen bereitstellt. Dieses Forschungsfeld nimmt - anders als etwa die sozialwissenschaftliche Kommunikations- und Medienforschung, die weithin durch das Paradigma der Medienwirkung bestimmt ist und im Wesentlichen als geklärt voraussetzt, was Medien sind - gerade die Medialität der Medien und auch die des Mediums Sprache in den Blick, weil es die Frage nicht für theoretisch belanglos hält, was Medien sind (Münker/Roesler (Hg.) 2008) oder besser, wie Medien operieren: Medialität und Mentalität, Medialität und Performativität, Medialität und Theatralität (Buss et al. 2009); und auch wieder die Frage nach dem Verhältnis von Medialität und Zeichenhaftigkeit (Winkler 1997, 2008; Wirth 2008) tritt ebenso in den Fokus der Forschung wie der Bild-Sprach-Zusammenhang (Holly 2008), das Problem der Stimmlichkeit, der Schriftlichkeit oder der Raumsituiertheit von Sprache. Wie sichtbar sich dieses Forschungsparadigma, das in den letzten zehn Jahren nicht unwesentlich von einer Gruppe von kulturwissenschaftlichen DFG-Sonderforschungsbereichen etwa in Berlin, Köln, Siegen und Gießen konturiert worden ist, inzwischen etabliert hat, lässt sich auch daran ablesen, dass der Wissenschaftsrat in seinen jüngsten „Empfehlungen zur Weiterentwicklung der Kommunikations- und Medienwissenschaften in Deutschland“ (2007) in seiner Übersicht über das disziplinäre Feld neben den sozialwissenschaftlichen Kommunikationswissenschaften und der Medieninformatik als dritte Konstituente eine Wissenschaftsgruppe nennt, die er unter dem Titel „kulturwissenschaftliche Medialitätsforschung" zusammenfasst. Ich betrachte deshalb auch die diesjährige Jahrestagung des IDS als einen diskursiven Beitrag zur weiteren Profilierung einer kulturwissenschaftlichen Medialitätsforschung und insbesondere als einen Beitrag zur theoretischen Aufhellung des Begriffs der Medialität. Ohne Zweifel lässt sich der Begriff der Medialität - und deshalb ist der Tagungstitel glücklich gewählt - nicht ohne die Diskussion dessen klären, was unter ,Intermedialität ${ }^{`}$ und - wie ich hinzufügen möchte - ,Intramedialität verstanden werden soll. Meinem Beitrag zum Tagungsthema liegt deshalb die These zugrunde, dass ein angemessener Begriff von Medialität nur über die Analyse der Begriffe ,Intramedialität ${ }^{\star}$ und ,Intermedialität ${ }^{`}$ gewonnen werden kann, wobei ich - wie im Folgenden deutlich werden soll - beide als operative Begriffe verstehen will, das heißt als Begriffe, mit denen ein bestimmtes Verfahren der Medien beschrieben werden kann - das Verfahren der Transkription. ,Transkriptivität', der dritte Begriff, der im Folgenden eine Rolle spielen wird, soll dabei nicht nur mit Blick auf die Sprache, sondern darüber hinaus auf das Feld der kulturellen Semiosis insgesamt bezogen werden. 


\section{Kulturelle Semiosis und die ,Logik der Transkription}

Dass Medien intermedial aufeinander Bezug nehmen, dass sie sich intramedial in rekursiven Schleifen auf sich selbst beziehen, dass sie also ihre eigenen Hervorbringungen unablässig zum Ausgangspunkt von Resemantisierungen, von Um- und Überschreibungen machen, ist ein Phänomen, auf das vor allem in jüngerer Zeit in den unterschiedlichsten disziplinären Kontexten der Kulturwissenschaften wachsende Aufmerksamkeit gerichtet wird. In den Kunstwissenschaften sind Begriffe wie der der „Metakunst" bzw. der „metapeinture“ gängige Beschreibungskategorien für die Kunst des 20. Jahrhunderts: Stoichita (1993/1998) etwa hat es als ein Kennzeichen der modernen Malerei angesehen, dass sie selbstreferentiell geworden sei, und deshalb von einem ,autoreferentiellen Malereidiskurs“ der Moderne gesprochen, ${ }^{2}$ und Mitchell hebt in seiner Bild-Theorie hervor: „Selbst-Referenz ist ein zentrales Thema modernistischer Ästhetik und ihrer verschiedenen postmodernen Revisionen. ${ }^{\text {" }}$ Bezugnahmeformen dieser Art bestimmen auch andere Medien und kulturelle Praktiken: das Remake im Film, Appropriationen in der Fotografie 4 oder Cover-Hits und Samplings in der Unterhaltungsmusik. Die Musik insgesamt ist wie vielleicht keine andere Kunst durch Verfahren der bearbeitenden Selbstbezugnahme, durch Verfahren des Arrangements, der Kontrafaktur, der Parodie und Umorchestrierung, durch Revision, Variation und Improvisation ${ }^{5}$ geprägt. Auch Sprache scheint in geradezu paradigmatischer Form durch ihr Vermögen rekursiver Selbstbezugnahme bestimmt zu sein, durch das Vermögen, Ausschnitte der Rede gleichsam stillzustellen und sie - wie etwa Gülich/Kotschi oder Mar-

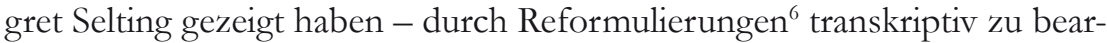
beiten, ${ }^{7}$ eine autoreferentielle Selbstlektüre, die Almuth Grésillion ${ }^{8}$ auch für das literarische Schreiben als bestimmendes operatives Moment herausgearbeitet hat.

\footnotetext{
Vgl. etwa Zuschlag (2002).

Vgl. hierzu Zuschlag (2002, S. 177).

Vgl. Mitchell (1995, S. 35): „Self-reference is a central issue in modernistic aesthetics and its various postmodern revisions."

4 Zum Problem des transkriptiven Verhältnisses von Original und Kopie in Fällen etwa des Samplings und der Appropriation Art vgl. Fehrmann/Linz/Schumacher/Weingart (2004). Zum Problem der Appropriationskunst vgl. etwa Amelunxen (1993) und Aigner (1993).

5 Vgl. Leopold (Hg.) (1992), hier Leopold (1992, S. 8); vgl. ebenso die Beiträge in Buschmeier/ Konrad/Riethmüller (Hg.) (2008).

6 Vgl. hierzu etwa Gülich/Kotschi (1996).

7 Mit transkriptiven Bearbeitungen sind selbstexplikative und kooperative Verständigungshandlungen gemeint, wie sie etwa in der Ethnomethodologie untersucht worden sind: Reparatur-Sequenzen, ,in denen ein Interaktionspartner - oft in einer Nebensequenz [...] eine vorausgegangene Äußerung korrigiert oder präzisiert oder ein vom Rezipienten der Äußerung signalisiertes Verstehensproblem bearbeitet“ (Selting 1987, S. 128); vgl. hierzu insgesamt Jäger (2004).

$8 \quad$ Vgl. Grésillion (1995, S. 8, Anm. 9).
} 
Auch jenseits der Selbstreferentialität des Schreibaktes lassen sich - etwa in Kristevas (1969) Begriff der „Intertextualität“ oder in Genettes (1993, S. 9) Kategorie der „Transtextualität“ - literarische Bezugnahmeformen ausmachen, durch die Texte ,in eine manifeste oder geheime Beziehung zu anderen Texten" (ebd.) gebracht und so die Verwebungsdichte generiert wird, durch die literarische Universen bestimmt sind.

Schließlich lässt sich auch im Zeitalter der, neuen Medien' - so Bolter und Grusin ${ }^{9}$ - die Logik der intermedialen Verhältnisse als eine Logik der „Remediatisierung“ („remediation") beschreiben: Medien treiben - so Manovich - sowohl auf der Inhalts- als auch auf der Formebene durch Übersetzung, Umgestaltung und Umformung von anderen Medien die Prozesse der „kulturellen Rekonzeptualisierung“ voran. ${ }^{10}$

\section{Transkriptivität: Zeichen- und erkenntnistheoretische Sondierungen}

Hinter der hier nur angedeuteten Vielfalt der „Praktiken der Selbst- und Fremd-Bezugnahme" ${ }^{11}$ die die Verfahren der Sprache, der Künste, der Kommunikationsmedien ${ }^{12}$ und der neuen Medien ${ }^{13}$ - und damit wesentliche Teile der kulturellen Semiosis - bestimmen, lässt sich nun - so unterschiedlich sie auch in medialer und ästhetischer Hinsicht ausgeprägt sein mögen - eine grundlegende Verfahrenslogik ausmachen, die ich in verschiedenen Arbeiten vorgeschlagen habe, als eine ,Logik der Transkription zu verstehen (vgl. Jäger 2008a). In alle diese Verfahren der intra- und intermedialen Kopplung und Bezugnahme ist - so meine These - eine allgemeinere mediale Operationslogik eingeschrieben, die für die Sprach-, Schrift- und Bildmedien ebenso gilt wie für die sogenannten ,neuen Medien': Für alle diese Medien- und Zeichensysteme ist es charakteristisch, dass sie ihre sinngenerierenden und sinntransformierenden Leistungen we-

9 Bolter/Grusin (2001, S. 45): „[W] call the representation of one medium in another remediation, and will argue that remediation is a defining characteristic of the new digital media." Ebenso etwa S. 55: „It would seem, then, that all mediation is remediation. [...] No medium, it seems, can now function indepedently and establish its own seperate and purified space of cultural meaning. “

10 Vgl. Manovich (2001, S. 47): „In new media lingo, to 'transcode' something is to translate it into another format. The computerization of culture gradually accomplishes similar transcoding in relation to all cultural categories and concepts. That is, cultural categories and concepts are substituted, on the level of meaning and/or language, by new ones that derive from the computer's ontology, epistemology, and pragmatics. New media thus acts as a forerunner of this more general process of cultural reconceptualization."

11 Vgl. zu diesen „Praktiken des Sekundären“ auch insgesamt Fehrmann/Linz/Schumacher/ Weingart (Hg.) (2004).

12 Vgl. hierzu etwa Luhmann (1997a, S. 190-412).

13 Vgl. etwa Manovich (2001). 
sentlich in semiologischen Prozeduren der inter- und intramedialen Bezugnahme organisieren. Diese Prozeduren sollen im Folgenden am Leitfaden von fünf heuristischen Prinzipien zur intra- und intermedialen Bezugnahme näher in den Blick genommen werden.

\subsection{Das Spur-Prinzip ${ }^{14}$}

Für das Modell der Intra- und Intermedialität, das ich hier skizzieren möchte, ist die Maxime Brandoms zentral, dass die Fähigkeit von Subjekten, mit Zeichen auf Gegenstände einer transsemiotischen Welt Bezug zu nehmen, in Begriffen der Fähigkeit erklärt werden muss, mit Zeichen auf Zeichen Bezug zu nehmen. Man könnte diese Maxime auch so formulieren, dass ,in der Reihenfolge semantischer Erklärungen der Inferenz Vorrang vor der Referenz eingeräumt werden muß“ (Brandom 2001, S. 9). Mit dieser Priorisierung inferentieller vor referentiellen Bezugnahmen bindet Brandom den repräsentationalen Gehalt von Begriffen und Behauptungen, kurz die interne Sphäre des Mentalen an den externen Raum medialer Diskursivität. ${ }^{15}$ Sein Begriff der ,expressiven Vernunft“ lässt sich insofern auch als der einer diskursiven oder - allgemeiner - medialen Vernunft verstehen: Für Brandom sind kognitive Operationen als mentale Prozesse, die referentielle Bezugnahmen ermöglichen, auf die expressive Spur ihrer medialen Erscheinung angewiesen, weil sich erst hier, im medialen Raum kommunikativer Bezugnahmen, sowohl Subjekte möglicher Referenzhandlungen als auch Welten ausbilden können, auf die referiert werden kann.

Brandoms Privilegierung inferentieller Bezugnahmen (vor referentiellen) - von der bei der Erörterung des Interpretations-Prin₹ips noch näher die Rede sein wird - muss im Horizont einer zugleich epistemologischen und semiologischen Grundannahme gelesen werden, die sich in der sprachphilosophischen Kritik des frühen 19. Jahrhunderts an der cartesianisch-kantischen Subjekt- und Sprachidee ausgebildet hat. Der Kern dieser Hypothese lässt sich so formulieren, dass in dem Tripel ,Erkenntnissubjekt - Zeichen - Erkenntnisobjekt ${ }^{\star}$ das Zeichen insofern eine zentrale Rolle spielt, als es eine notwendige Entstehungs- und Bestandsbedingung für die beiden anderen Konstituenten darstellt. Sowohl die begriffliche Ausdifferenzierung der Welt als auch die Herausbildung des Bewusstseins, das sich auf sie bezieht, sind ohne den medialen ,Umweg' semiologischer Selbstlektüre und zeichenvermittelter Interaktion mit anderen, d.h. ohne intra- und intermediale Bezug-

14 Vgl. zum Spur-Begriff etwa die Arbeiten in Fehrmann/Linz/Epping-Jäger (Hg.) (2005); ebenso Krämer/Kogge/Grube (Hg.) (2007).

15 Vgl. Brandom (2001, S. 207 ff.); vgl. etwa auch Brandom (2000, S. 399 f.): „Die für uns charakteristische Verstandesfähigkeit ist ein Status, der im Rahmen einer Struktur wechselseitigen Anerkennens gewonnen wird [...]. Der spezifisch diskursive Charakter dieser normativen sozialen Struktur [...] besteht in der inferentiellen Gliederung dieser Anerkennungspraktiken. Wir sind diejenigen, die Gründe für das, was wir sagen und tun, geben und verlangen.“ 
nahmen, nicht möglich. Erst auf dem Umweg einer semiologisch vermittelten Selbstlektüre - die zugleich in ein komplexes Netzwerk kultureller Texturen eingewoben ist - kann das Subjekt sich in seiner Erkenntnisbeziehung zur Welt konstituieren. ${ }^{16}$ Man könnte mit Blick auf unser Thema auch sagen, dass sich in gewissen Sinne Medialität in die Unmittelbarkeit einnistet, in der sich das Subjekt auf sich selbst und seine Erkenntniswelt bezieht.

Die Figur der zeichenvermittelten und bewusstseinskonstitutiven Rückwendung des Subjektes auf sich selbst hatte zum ersten Mal Humboldt in seiner Sprach- und Zeichenphilosophie entfaltet. Gegenüber der cartesianisch-kantischen Subjektidee machte er geltend, dass die ,innerliche intellectuelle Thätigkeit“ eines solipsistischen Subjektes ,gewissermassen spurlos vorübergehend“" (Humboldt GS 7, S. 53) wäre, wenn sie sich nicht an die Äußerlichkeit des Lautes in der Rede binde. Ohne eine solche mediale Vermittlung des Mentalen könne - so Humboldt (ebd.) - „das Denken [...] nicht zur Deutlichkeit gelangen, die Vorstellung nicht zum Begriff werden“. Die Äußerlichkeit der Rede, der performative Auftritt der Zeichen im Horizont von Zeichennetzwerken, ist für Humboldt jener mediale Ort, an dem allein es möglich wird, „, den Gedanken [...] zur Rückwirkung auf das Subject, aus sich heraus und sich gegenüber zu stellen" (Humboldt GS 5, S. 455). „Ohne diese, wo die Sprache mitwirkt, [...] immer vorgehende Versetzung in zum Subject zurückkehrende Objectivität ist die Bildung des Begriffs, mithin alles wahre Denken unmöglich.“ (ebd., S. 377) $\cdot{ }^{17}$ Erst in der Spurenlese, der Relektüre, in der der ,Geist" der medialen Spur der eigenen mentalen Akte begegnet, in der Transkription des Mentalen in die semiologischen Register des Medialen, kann sich begriffliche Distinktivität einstellen und ein Subjekt möglicher begrifflicher Unterscheidungshandlungen konstituieren.

Bereits Humboldt entwirft dabei die medial vermittelte autoreferentielle Selbstbegegnung des Geistes als ein Verfahren, in dessen Vollzug der vorgängige Begriff im Akt seiner Bezeichnung in gewissem Sinne erst hervorgebracht wird: Die Selbstlektüre des Geistes nimmt die Form einer ,Begriffsbezeichnung' an, in der - wie Humboldt (GS 5, S. 436) formuliert - „die Bezeichnung erst das Entstehen des zu Bezeichnenden vor dem Geiste vollendet". Das Vorgängige, das zu Bezeichnende, wird erst in der Nachträglichkeit der Bezeichnung, gleichsam in einer transkriptiven Umschrift, konstituiert.

16 Obwohl das epistemologische Modell der Semiologie des auslaufenden 19. Jahrhunderts nicht direkt auf Bruno Latours Actor-Network-Modell abgebildet werden kann - so müssen etwa bei Latour ,actors' nicht notwendig , human actors' sein -, stimmen doch beide Ansätze in der Bestimmung des Akteur-Netzwerk-Verhältnisses überein: „No net exists independently of the very act of tracing it, and no tracing is done by an actor exterior to the net." (Latour 1996, S. 378).

17 Vgl. auch Humboldt (GS 6, S. 155 und GS 7, S. 55); vgl. hierzu ausführlich Jäger (1988). 
Man könnte auch, obgleich für Derrida wohl zuletzt Humboldt als Bürge des Gedankens in Frage käme, eine solche spurtheoretische Überlegung mit Derrida in Verbindung bringen. Wie vor ihm Humboldt skizziert Derrida die theoretischen Umrisse einer - wie man sie nennen könnte - transzendental-medialen Bedingung von Mentalität, die gegen die Metaphysik der „Innerlichkeit der Seele“" (Derrida 1974, S. 61) das Draußen exteriorer Zeichenprozesse in Stellung bringt: Er wendet sich insbesondere gegen die Idee eines „transzendentalen Signifikats“ als „Ausdruck reiner Intelligibilität“ (ebd., S. 28), gegen die Metaphysik der „Selbstpräsenz der Seele im wahren Logos" (ebd., S. 61)..$^{18}$ In einem gleichsam medientheoretischen Gestus insistiert er gegenüber den klassischen Zeichen- und Bewusstseinsphilosophien auf der grundlegenden Bedeutung des Signifikanten für die Konstitution des Signifikats; die Vorgängigkeit des Zeichensinns und des Bewusstseins, das ihn erzeugt, lässt sich immer nur auf dem Weg der nachträglichen Prozessierung durch materielle Zeichen herstellen; die Privilegierung des Signifikats ist illegitim, weil ein Bezeichnetes unabhängig von der Phänomenalität des Zeichens nicht existent sein könnte: „Es gibt [...] keine Phänomenalität, welche das Zeichen oder den Repräsentanten reduziert, um schließlich das bezeichnete Ding im Glanz seiner Präsenz erstrahlen zu lassen.“ (Derrida 1974, S. 86).

Derridas Angriff richtet sich also - wie zuvor der Humboldts - auf die Metaphysik der Präsenz, die Idee der unmittelbaren „Selbstpräsenz des cogito" (ebd., S. 26) und seiner signifikativen Leistungen, indem er die Untilgbarkeit des medialen „Aufschubs“ ${ }^{19}$ der sich der „Exteriorität des Signifikanten“ (ebd., S. 29) bedienen muss, exponiert, eines medialen Aufschubs, der für den Prozess der Selbstkonstitution des Geistes und seiner Sinnproduktionen absolut unabdingbar ist. Die Vorgängigkeit des Sinns ist deshalb - ebenso wie das mentale System, das ihn generiert - für Derrida immer notwendig auf die Nachträglichkeit einer medialen Spur der eigenen Aktivität verwiesen: Das einfache „Frühersein der Idee“ oder der ,inneren Absicht" gegenüber ihrer medialen Prozessierung ist ein Vorurteil (vgl. Derrida 1976, S. 24). Mit Freud betrachtet er die ,Umschrift' des Unbewussten in einen Bewusstseinstext nicht als einen Prozess der nachträglichen ,Übersetzung' eines ,Ursprünglichen': Vielmehr ist bereits der ,ursprünglich` unbewusste Text

aus Archiven gebildet, die immer schon Umschriften sind. [...] Alles fängt mit einer Reproduktion an. ,Immer schon' heißt Niederschlag eines Sinns, der nie gegenwärtig war, dessen bedeutete Präsenz immer ,nachträglich', im Nachherein und zusätzlich rekonstruiert wird. Das Aufgebot des Nachtrags ist hier ursprünglich und untergräbt das, was man nachträglich als Präsenz rekonstruiert. (Derrida 1976, S. 323)

18 Dass hierbei nach Derridas Ansicht immer die Stimme als Komplize der reinen Selbstaffektion auftritt, soll hier aus den dargelegten Gründen ausgeblendet werden.

19 Vgl. hierzu etwa Derrida (2003, S. 118 ff.). 
Die Umschrift, die Transkription, vollzieht sich in der Nachträglichkeit einer Bezeichnung, die erst - wie Humboldt formuliert hatte - das vorgängig zu Bezeichnende vor dem Geiste vollendet.

Sinn lässt sich also für Derrida wie für Freud und Humboldt „,nicht in der ursprünglichen oder in einer modifizierten Form der Präsenz denken. “ (Derrida 1976, S. 323). Im „Spiel des Bezeichnens“ wird mit dem Signifikanten kein vorgängiger Sinn substituiert, weil dieses Zeichen-Substitut so Derrida (ebd., S. 424) - nichts ersetzt, „das ihm irgendwie präexistiert hätte." Sinn ist - wie man in Husserlscher Terminologie sagen könnte - in seiner semantischen Evidenz nicht in ursprünglicher „Selbsthabe“, sondern als Ergebnis der diskursiven Leistung einer nachträglichen „Selbstgebung “ kurz: allein als das Ergebnis einer transkriptiven Umschrift, einer zugleich nachträglichen und für das Vorgängige konstitutiven semiologischen Bezugnahme gegeben. „Der Gehalt des Geistes“ - formuliert Cassirer (1964, S. 18) „erschließt sich nur in seiner Äußerung“"

Am Ende meines kleinen spurtheoretischen Exkurses lässt sich also mit Blick auf den zeichen- und erkenntnistheoretischen Rahmen einer Theorie der Intermedialität als erster Befund festhalten: Transkriptive Bezugnahmen sind mediale Verfahren der Konstitution des Mentalen: Sie sind der operative Modus, in dem der Geist sich selbst in Figuren der medialen Nachträglichkeit auf die Spur kommt. Diese suspendieren gleichsam die Unmittelbarkeit des Selbst- und Weltbezugs, in die sie sich als mediale Verfahren einschreiben.

\subsection{Das Interpretations-Prinzip}

Dass die kulturelle Semiosis der systematische Ort ist, an dem Zeichensubjekte ihre mentale Identität über die Spur medialer Zeichenhandlungen konstituieren, verweist auf eine weitere wesentliche epistemologisch-semiologische Voraussetzung einer Theorie intra- und intermedialer Transkriptivität: Die Semiosis ist nämlich nicht nur das mediale Feld, auf dem Subjekte sich als Subjekte konstituieren, sondern uno actu zugleich die semiologische Bühne, auf der sich kulturelle Welten in ihrer begrifflichen Ordnung herstellen, jene Welten also, in denen und in Berug auf die die Subjekte handeln. Eine prämediale Welt möglicher Bezugnahme bzw. eine prämediale ,Sprache des Geistes' kann es dann ebenso wenig geben wie Subjekte, die ihren medialen Zeichenhandlungen als selbstpräsente Cogitos vorauslägen.

Für die Genese der kulturellen Semantik heißt dies: Die Konstitution und Beglaubigung von Sinn lässt sich nicht auf dem Wege der jeweiligen referentiellen Abgleichung von Zeichensystemen mit einer medientranszendenten Realwelt bzw. ihren kognitiven Repräsentationen vollziehen. Vielmehr kann die semantische Ratifizierung von Sinn ihren Ort zum einen 20 Vgl. Husserl (1929, S. 140 ff.). 
nur innerhalb des Horizontes von und zum anderen nur in wechselseitiger Bezugnahme zwischen medialen und semiologischen Darstellungssystemen haben. Eine „beobachtungsinvariante Welt“ steht - wie Luhmann (1992, S. 75) bemerkt - für die Prüfung des Adäquationsgrades semiologischer Darstellungssysteme nicht zur Verfügung: ,[D]ie Welt kann nicht von außen beobachtet werden, sondern nur in ihr selbst, das heißt: nur nach Maßgabe von [...] Bedingungen, die sie selbst bereitstellt" (ebd.).

Die Geltung und die semantische Evidenz von kulturellem Sinn, wie er von sprachlichen und nichtsprachlichen Medien generiert wird, verdanken sich deshalb einem Prinzip, das man Interpretations-Prinzip nennen könnte und das zugleich ein Intermedialitäts-Prinzip ist. Das referentielle Verwenden von Zeichen zur Bezugnahme auf eine (zeichentranszendente) Welt ist kein denkbares Fundament für die sinnkonstitutiven Leistungen von Zeichensystemen. ${ }^{21}$ Aus einer ontologischen Weltwabe lässt sich ebenso wenig semantischer Honig saugen wie aus den mentalen Leistungen eines vorsprachlichen Geistes. Sprach- und Mediensysteme, die auf eine semantische Referenzfunktion eingeschränkt blieben, könnten keine Sprach- oder Mediensysteme im definitorischen Sinne sein.

Was in einer paradigmatischen Weise für natürliche Sprachen gilt, scheint eine Eigenschaft von Mediensystemen insgesamt zu sein: dass sie nämlich in einem für sie konstitutiven Sinne über die Möglichkeit verfügen müssen, die semiologischen Mittel, mit denen ihre Verwender interagieren und durch die sie sich auf die Welt beziehen, jederzeit als solche Mittel zu fokussieren, zu thematisieren und sie im Interesse der Selbst- und Fremdverständigung in autoreferentiellen oder interaktiven semiologischen Aktivitäten semantisch zu bearbeiten. ${ }^{22}$ Die Fähigkeit, selbst hervorgebrachte oder kommunikativ vorgefundene symbolische Artefakte zu zitieren, zu paraphrasieren, zu explizieren, zu erläutern oder zu interpretieren, um auf diese Weise ihren

21 Vgl. hierzu Saussures Überlegungen in den Gartenhausnotizen: Gegen die Annahme, dass man bei Worten, die sich „,auf einen materiellen Gegenstand“ beziehen, sagen könnte, „daß das Wesen des Gegenstandes selbst tatsächlich so beschaffen ist, daß es dem Wort eine positive Bedeutung gibt“, wendet er ein: „Ich kenne tatsächlich keinen Gegenstand, zu dessen Bezeichnung nicht einer oder mehrere angeblich zusätzliche Begriffe hinzukommen, die aber im Grunde ganz genauso wichtig wie der Hauptbegriff sind - selbst wenn der fragliche Gegenstand die Sonne, das Wasser, die Luft, der Baum, die Frau, das Licht usw. wäre. Derart, daß alle diese Bezeichnungen in Wirklichkeit ebenfalls negativ sind und nur im Verhältnis zu den Begriffen etwas bedeuten, die in anderen ebenfalls negativen Termen enthalten sind, derart, daß sie sich niemals auf einen in sich bestimmten Gegenstand beziehen wollen und in Wirklichkeit diesen Gegenstand [...] nur auf indirekte Weise, durch und unter der Perspektive dieses oder jenes besonderen Begriffs berühren“" (Saussure 2003, S. 141 f.).

22 Vgl. Brandom (2001, S. 210 f.): „Auf der Seite der propositional gehaltvollen Sprechakte [...] schlägt sich die wesentliche inferentielle Gliederung des Propositionalen in der Tatsache nieder, daß das Spiel des Gebens und Verlangens von Gründen das Herzstück der spezifisch sprachlichen Praxis ist. [...] Ansprüche bzw. Behauptungen dienen als Gründe und bedürfen ihrerseits der Begründung oder Rechtfertigung. Sie verdanken ihre Gehalte teilweise der Rolle, die sie in einem Netzwerk von Inferenzen spielen.“ 
Verwendungssinn fortzuschreiben, muss als ein Vermögen angesehen werden, das für das operative Wissen der Akteure konstitutiv ist, die sich in der Sphäre des Medialen bewegen.

Ehe es also Medienakteuren möglich ist, mit Zeichen auf die Welt zu referieren, muss das Spiel der Bezugnahme von Zeichen auf Zeichen möglich sein, müssen die Bedeutungen von Zeichen in dem Sinne verfügbar sein, dass ihre Vernetzung mit anderen Zeichen im System einer Sprache oder eines nichtsprachlichen Mediensystems aufgerufen, also das semantische Netzwissen ${ }^{23}$ bei Bedarf transkriptiv in Bezugnahmehandlungen aktiviert werden kann.

Die repräsentativ-referentielle Zeichen-Welt-Relation wäre in sich völlig unzureichend für die Gewährleistung semantischer Bezugnahmen, weil die referentielle Bezugnahme die Möglichkeit der zeichensystem-inhärenten Bezugnahme der Zeichen aufeinander - also das Spiel intra- und intermedialer Bezugnahmen - voraussetzt: „Zeichen referieren“ - so Deacon (1997, S. 99) - ,nicht direkt auf die Gegenstände in der Welt, sondern indirekt dadurch, dass sie auf andere Zeichen verweisen" [diese und die folgenden Übersetzungen von mir, L.J.]. Die medienimmanente Genese des Sinns folgt also einem semiotischen Gesetz, das Peirce (1984, S. 173) so formuliert hat: „Aus der Tatsache, dass jeder Gedanke ein Zeichen ist, folgt, dass der Gedanke einen weiteren Gedanken adressieren muß, weil darin das Wesen des Zeichens besteht. [J]eder Gedanke muss durch einen anderen Gedanken interpretiert worden sein." Es gibt - so Peirce (ebd., S. 224) „keine Ausnahme von dem Gesetz, dass jedes Gedanken-Zeichen durch ein folgendes übersetzt oder interpretiert worden sein muss [...].“

Für die Sprache etwa heißt dies: Mit sprachlichen Ausdrücken auf die Welt Bezug nehmen zu können, setzt voraus, dass die semantischen Gehalte der sprachlichen Äußerungen, mit denen Sprecher einen solchen Bezug herstellen, in ihrer Implizitheit expliziert, in ihrer Unklarheit erläutert, in ihrer Unverständlichkeit paraphrasiert sowie gegenüber Zweifeln legitimiert und begründet werden können etc., setzt also voraus, dass die Sprecher in einen Diskurs transkriptiver - in gewissem Sinne interpretierender - Bezugnahme auf ihren eigenen Sprachgebrauch einzutreten vermögen. ${ }^{24}$ Ein solches transkriptives Vermögen ist ein Grundprinzip der Pro-

23 Vgl. hierzu de Saussures Theoriefragment ,Notes Item', in dem er die Netzverwobenheit sprachlicher Zeichen theoretisch entfaltet (Saussure 1974, S. 35 ff. (3306-3324 = N 15.1-19)); vgl. hierzu Jäger (1986).

24 Das Verfügen über begriffliche Gehalte, das eine zentrale Voraussetzung der Bezugnahme auf transsemiotische Sachverhalte darstellt, ist - so Brandom (2000, S. 26) - eine ,sprachliche Angelegenheit, nicht in dem Sinn, daß man sprechen muß, um es zu tun, sondern in dem Sinn, daß man ein Mitspieler im wesentlich sprachlichen Spiel des Gebens und Verlangens von Gründen sein muß, um [zum Verfügen über begriffliche Gehalte] in der Lage zu sein“. Brandom geht hier davon aus, „daß die Eigenschaft, über propositionale Gehalte zu verfügen, anhand der Praktiken des Lieferns und Forderns von Gründen zu verstehen [ist]. Eine 
zessierung von kultureller Semantik. Es gilt in einer sicher noch näher zu untersuchenden Weise auch für andere Medien- und Zeichensysteme - für bestimmte Formen des Piktoralen etwa oder die Musik - und insbesondere für deren intermediales Zusammenspiel.

Semantiken verdanken sich also epistemologisch nicht einem Reich medienfreier Kognition oder einer ontologischen Ordnung der, Welt selbst ${ }^{6}$. Ihre Funktion kann sich in den verschiedenen Medien nicht darin erschöpfen, Weisen bereitzustellen, in denen eine prämediale (,ontologische) Welt dargestellt, abgebildet, gespiegelt zu werden vermag. Wir verfügen - so Rorty (1994, S. 321) - über keinen „transzendentalen Standpunkt außerhalb unserer gegenwärtigen Darstellungssysteme, von dem aus wir die Relation zwischen diesen Darstellungen und ihrem Gegenstand untersuchen könn $[t]$ en", über keinen exzentrischen archimedischen Punkt also, der es uns erlaubte, die Adäquatheit unserer Bezugnahmen auf die Welt unabhängig von medialen Darstellungssystemen zu beurteilen. Wir müssen uns vielmehr immer auf Semantiken stützen, für die das Interpretations-Prin₹ip konstitutiv ist. Semantiken dieses Typs verdanken sich Zeichensystemen, die es in einem gewissen Sinn nur erlauben, weitere semiologische Eintragungen in eine immer schon semiologisch strukturierte Welt vorzunehmen. ${ }^{25}$

Die Genese, Fortschreibung und Geltungsauszeichnung von Sinn operiert also über verschiedene Arten der Bezugnahme, die sich in einem erkenntnistheoretischen Sinn nicht vorgängig zwischen Zeichensystemen und der Welt abspielen, ${ }^{26}$ sondern die sich prioritär einmal zwischen verschiedenen (medialen) Zeichensystemen - also intermedial - und zum zweiten auch innerbalb desselben Zeichensystems - also intramedial - vollziehen. Transkriptionen sind die grundlegenden Verfahrensformen, die auf beiden Ebenen der Bezugnahme die kulturelle Semantik in Bewegung halten (vgl. Jäger 2008a). Dies ist der zweite Befund, den ich mit Blick auf den zu skizzierenden Rahmen einer Theorie der intra- und intermedialen Transkrip-

zentrale Behauptung lautet, daß diese Praktiken als soziale Praktiken zu verstehen sind - ja als sprachliche." (Brandom 2000, S. 219).

25 Natürlich müssen Sprachen, wie Peirce gezeigt hat, über Mittel verfügen, die es erlauben, die ,wirkliche“ Welt von einer, imaginären“ Welt zu unterscheiden: „Die wirkliche Welt kann von einer imaginären Welt nicht durch irgendeine Beschreibung unterschieden werden. Daher sind Pronomina und Indices notwendig" (CP 3.363, hier zitiert nach Peirce 1970, S. 104); vgl. hierzu auch Pape (1999, S. 7). Allerdings gilt auch - wie Brandom im Anschluss an Sellars gezeigt hat - für indexikalische Sätze, „daß sogar nichtinferentielle Berichte inferentiell gegliedert sein müssen“ (Brandom 2001, S. 70). ,[W] esentlich indexikalische Überzeugungen [besitzen] zwar eine besondere Art des auf Gegenstände bezogenen Gehalts, den andere Überzeugungen nicht haben, allerdings sollte man sich diese Gegenstandsbezogenheit nicht als nichtbegriffliches Element ihres Gehaltes vorstellen; vielmehr ist die besondere Art des $\mathrm{Zu}$ gangs zu jenen Gegenständen [...] eine besondere Art des begrifflich gegliederten Zugangs“" (Brandom 2000, S. 765). Brandom hält deshalb den intralingualen Bezug (Wort-Wort-Bezug) für „basaler“ als den Wort-Welt-Bezug (vgl. Brandom 2000, S. 442).

26 Natürlich kann man mit Zeichensystemen auf die Welt Bezug nehmen, aber nicht auf eine, die ohne Zeichensysteme strukturiert wäre. 
tivität festhalten möchte. Der Suspendierung des unmittelbaren Bezugs des Subjektes auf sich selbst, das des ,Umwegs' symbolischer Entäußerungen, einer medialen Spur seiner mentalen Aktivität bedarf, entspricht eine zweite konstitutive Suspendierung, nämlich die der Möglichkeit des unmittelbaren Bezugs auf eine Welt möglicher Referenz. Aus der Idee der Transkriptivität folgt also eine doppelte Suspendierung erkenntnistheoretischer Unmittelbarkeit, an deren Ort die Prozeduren medial vermittelter Selbst- und WeltBezugnahme treten.

\subsection{Das Medialitäts-Prinzip}

Spur-Prinzip und Interpretations-Prinzip schieben also unabweislich die Mittelbarkeit des Medialen in das Feld epistemologischer Unmittelbarkeit ein. Die Idee der Transkriptivität rehabilitiert die in der aristotelisch-kantischen Tradition der Sprach- und Zeichentheorie ebenso wie in ihren rezenten Ausläufern, etwa der kognitivistischen Linguistik, ignorierte und ausgeblendete „Materialität der Kommunikation“, ${ }^{27}$ und das heißt, sie setzt Materialität, Performativität und Äisthetik von Zeichen- und Mediensystemen theoretisch wieder in ihr Recht.

Wenn es zutrifft, dass Zeichen im Zuge der Semiosis weder unmittelbar auf den Attributreichtum einer zeichentranszendenten Welt noch unmittelbar auf die mentalen Hervorbringungen einer vorsprachlichen,Sprache des Geistes' zurückgreifen können, werden sie selbst zum Diskursort der Sinnproduktion: Ihre Funktion kann dann nicht mehr auf die der Repräsentation, des Transports bzw. der Übertragung von Inhalten beschränkt werden, weil sie konstitutiv an der Genese dieser Inhalte beteiligt sind. Was übertragen wird, muss zunächst - um einen Terminus Humboldts zu verwenden ,ausgemittelt ${ }^{\natural}$, d.h. in semiologischen Bezugnahmehandlungen konstituiert worden sein, und es kann jederzeit in störungsinduzierten kommunikativen Anschlusshandlungen aufgehoben, dementiert, verschoben oder affirmiert - kurz: transkribiert - werden. Die Genese von Sinn ist insofern eng mit der Medialität der Zeichensysteme verschaltet, in denen er hervorgebracht wird.

In der Tat sind in sprachlichen und nicht-sprachlichen Medien die jeweiligen Arten von Bedeutungsgehalten eng mit den jeweiligen materiellen Zeichensubstraten verknüpft; sie liegen diesen nicht als ,neutrale kognitive Formen voraus. ${ }^{28}$ Die Annahme, dass - wie etwa noch Wittgenstein im

27 Vgl. Gumbrecht/Pfeiffer (Hg.) (1988).

28 Es gibt - wie Saussure für die Sprache formuliert - „keine positive Trennlinie zwischen dem Begriff des Wortes und dem Begriff des Begriffs, der im Wort ist" (Saussure 2003, S. 151); Saussure insistiert darauf, dass ,weder der Begriff noch das Zeichen, weder die Verschiedenheit der Zeichen noch die Verschiedenheit der Begriffe jemals für sich genommen einen gegebenen Term ausmachen: Gegeben ist nur die Verschiedenheit der Zeichen, die unauflöslich und auf unendlich komplexe Weise mit der Verschiedenheit der Begriffe verbunden ist. Indem sich diese beiden chaotischen Bereiche [les deux chaos] vereinigen, entsteht eine Ordnung." (ebd., S. 114; vgl. ebenso S. 136). 
Tractatus (im Anschluss an ein aristotelisches Modell von Mentalität und Medialität) annimmt - sich die logische Form eines Gedankens identisch sowohl im Gedanken als auch in einem gesprochenen oder geschriebenen Satz, der den Gedanken zum Ausdruck bringt, repliziert, muss nun zurückgewiesen werden. Unter den Bedingungen der Transkriptivität kann es die identische Replikation eines, kognitiven Originals' in verschiedenen Zeichenformaten nicht geben. Wir können - wie Danto (1995, S. 134) formuliert - nicht mehr voraussetzen, „daß dieselbe Form sozusagen in drei verschiedenen Medien verkörpert wird, dem Medium des Gedankens, des Schreibens und des Sprechens". Die Semantik etwa der Bilder - oder anderer nichtsprachlicher Medien - besteht deshalb auch nicht darin, dass sie etwas bildlich sagen, was auch sprachlich oder anders hätte gesagt werden können. Neutrale Inhalte bzw. Informationen, die gleichsam unversehrt (,originaliter') zwischen verschiedenen Medien übertragen werden können, sind nicht denkbar, weil es nur mediale Varianten von Inhalten gibt, für die kein prämediales Original existiert. Jede Form der Übertragung eines Inhaltes aus einem in ein anderes Medium nimmt deshalb notwendig die Form der Transkription, d.h. der Neukonstitution unter medial veränderten Bedingungen an.

Wie meine bisherigen Bemerkungen deutlich machen sollten, führen die theoretischen Konsequenzen, die sich aus dem Spur-Prinzip und dem Interpretations-Prinzip ergeben, zu einem Medienbegriff, der mit der Auffassung, Medien seien technische Mittel der Informationsübertragung, nicht kompatibel ist. Ein solcher Übertragungs-Begriff ${ }^{29}$ von ,Medium ' ist ohne Zweifel weder für technische noch für semiologische Medien und insbesondere nicht für das Medium Sprache angemessen. Die semantischen Gehalte von Zeichen gehen ihrer Übermittlung durch Zeichenausdrücke nicht als kognitives Reservoir voraus. Sie werden nicht als transzendente Signifikate in den Diskurs eingespeist, sondern dieser ist der generische Ort der Hervorbringung von Sinn. Die von der Epistemologie der Störung bewirkte doppelte Suspendierung der Unmittelbarkeit (die der Introspektion und der Referens) ist also zugleich mit einer Rehabilitierung medialer Mittelbarkeit verbunden.

\subsection{Das Rekursions-Prinzip}

Ein wesentlicher Verfahrensmodus von symbolisch-medialen Systemen besteht - wie bereits bei der Betrachtung des Spur- und der InterpretationsPrinzips deutlich geworden ist - in der transkriptiven Wiederverarbeitung von medialen bzw. symbolischen Hervorbringungen, die bereits zuvor in diesen Systemen generiert worden waren: „, [A]lle Medien hängen“ - wie Bolter/Grusin formulieren - „in Zyklen der Remediatisierung von anderen

29 Dass der Begriff der ,Übertragung‘ jenseits von Sender-Empfänger-Modellen der Kommunikation medientheoretisch angemessen rekonstruiert werden kann, hat Hartmut Winkler gezeigt; vgl. etwa Winkler (2004). 
Medien ab [...].“30 Sowohl Individuen als auch kulturelle Systeme organisieren den semantischen Prozess wesentlich über Formen der autoreferentiellen, intra- und intermedialen Selbstverarbeitung, der rekursiven Rückwendung des Systems auf sich selbst: Es ist diese Rekursivität, die - wie Luhmann formuliert ${ }^{31}$ - „Anwendung des Verfahrens auf die Resultate des Verfahrens “32, also etwa die Anwendung von Kommunikation auf die Resultate von Kommunikation, die ein „Eigenverhalten“, eine „Eigenwertproduktion" des Systems erzeuge.

Die Pointe dieses Luhmannschen Gedankens ${ }^{33}$ lässt sich im Hinblick auf Medien- und Kommunikationssysteme vielleicht so formulieren: Symbolische Systeme tendieren dazu, als Gewinn aus der für sie charakteristischen Verfahrensform der rekursiven Selbstverarbeitung Eigensinn ${ }^{34}$ zu generieren. Dies gilt insbesondere für Sprache, die in paradigmatischer Weise über die Eigenschaft verfügt, sich rekursiv auf sich selbst zurückzubiegen und so die eigene Zeichenverwendung fortlaufend zum Gegenstand weiterer thematisierender, kommentierender, explizierender oder zitierender Zeichenverwendungen zu machen, zum Objekt also selbstbezüglicher semiologischer Operationen, in denen sich das zeigt, was man die ,rekursive Transkriptivität‘ der Sprache nennen könnte.

Bereits die außerhalb des Verfahrensraumes der Schrift operierende Rede verfügt über das Vermögen der Isolierung und Stillstellung von Redeausschnitten sowie der Bearbeitung der stillgestellten ,Elemente und damit über eine Form des autoreferentiellen Bezuges von Sprache auf Sprache. ${ }^{35}$ Mit der Schrift entstehen dann neue Formen der intramedialen Bezugnahme, hypoleptische Bezugnahmen von Texten auf Texte, wie Raible (1995) ${ }^{36}$ sie genannt hatte, wobei auch noch für neue Digitalmedien die Remediation eines Mediums durch ein anderes als „defining characteristic“ angesehen werden muss - wie Bolter/Grusin (2001, S. 45) formulieren. Alle Stillstellungs-Artefakte unserer medialen Kulturen - Bilder, Texte, Partituren, Speicherungen

30 Vgl. Bolter/Grusin (2001, S. 55): ,[A]ll media depend on other media in cycles of remediation $[\ldots] . “$

31 Vgl. Luhmann (1997a, S. 217 f., S. 614, S. 888).

32 Vgl. Luhmann (1997, S. 213 f.): ,Er [der Begriff ,Eigenverhalten'] bezeichnet eine im rekursiven Verfahren der Anwendung des Verfahrens auf die Resultate des Verfahrens sich einstellende Stabilität.“

33 Ich möchte allerdings weder Luhmanns Medium/Form-Unterscheidung, noch deren Applikation auf Sprache übernehmen: Die These Luhmanns, Sprache sei ein „Nichtsystem“, das „Systembildungen im Bereich von Bewußtsein und Kommunikation erst ermöglicht", kann hier nicht diskutiert werden (vgl. Luhmann 1992, S. 51). Auch seine Behauptung, die Sprache sei kein System von Zeichen (ebd., S. 51 f.), beruht auf einer ziemlich unzulänglichen Kenntnis der Zeichentheorien etwa de Saussures, dem er eine abbildtheoretische Konzeption unterstellt.

34 Vgl. hierzu etwa Jäger (2005a).

35 Vgl. hierzu ausführlich Jäger (2005b).

36 Vgl. zum Begriff „Hypolepsis“ Assmann (1992, S. 282, S. 286 ff.). 
jedweder Art - dürfen so verstanden werden als Adressen möglicher Bezugnahmen, möglicher transkriptiver Adressierungen in den Archiven des kulturellen Gedächtnisses. Selbstberüglichkeit wäre also eine der grundlegenden Quellen kultureller Semantik, ein Verfahren, in dem in der Rückwendung eines symbolischen Systems auf sich selbst symbolische Artefakte aus ihren vorgängigen diskursiven Zirkulationsbedingungen gelöst und zur Bearbeitung bzw. Wiedereinfädelung in den semantischen Haushalt unter neuen Kontextbedingungen vorübergehend stillgestellt, zitiert, paraphrasiert, expliziert - kurz: de- und rekontextualisiert - werden.

Der vorübergehende oder länger anhaltende Zustand des Herausgelöstseins symbolischer Zeichenketten aus der Transitorizität ihrer Performanz ist insofern keine privilegierte Eigenschaft der Schrift oder anderer Stillstellungs-Artefakte, die die Verfahren der Medien ständig hervorbringen, sondern ein Durchgangsstadium in kommunikativen Prozessen, in dem mediale Entitäten eine vorübergehende oder dauerhafte ,Starre' derart annehmen, dass auf sie kommunikativ Bezug genommen werden kann, Sinn generiert und weiterverarbeitet zu werden vermag.

Unabhängig davon, ob die temporäre oder länger andauernde Stillstellung symbolischer Ketten die Form der Schrift annimmt oder die Form von in mündlichen Traditionen erstarrten „fixed texts “637 oder die der Paraphrase, der Explikation bzw. des Zitats in sprachlichen oder bildlichen Medien: In allen diesen Formen haben wir es mit einer rekursiven Alternierung von Stillstellung und intra- bzw. intermedialer Bewegung zu tun, die prinzipiell der Bearbeitung und Konstitution von Sinn im semantischen Haushalt von Kulturen zugrunde liegt. Transkriptive Verfahren, so lässt sich beim gegenwärtigen Stand unserer Überlegungen resümieren, sind jene Verfahren der remediatisierenden Bezugnahme von Symbolsystemen auf Symbolsysteme, durch die der Sinnkonstitutionsapparat der kulturellen Semantik als ein Apparat fortwährender De- und Rekonzeptualisierung in Gang gehalten wird. In die Konstitution von Sinn ist immer eine mediale Bewegung eingeschrieben, in deren Vollzug sich Medien in einer rekursiven Geste auf sich selbst oder auf andere Medien beziehen: Eine rekursionsfreie Konstitution von Sinn kann es nicht geben. Immer sind es Spuren vergangener Mediationen, auf die interpretierend in rekursiver Rückwendung Bezug genommen werden muss, damit in den Verfahren der kulturellen Semantik Sinn generiert werden kann.

\subsection{Das Störungs-Prinzip}

In gewissem Sinne können die bislang skizzierten Prinzipien als Voraussetzungen eines fünften Prinzips angesehen werden, das ich das Störungs-Prinzip nennen möchte. Wenn sich die Prozesse kultureller Sinnbildung not-

\footnotetext{
Vgl. hierzu Vansina (1973, S. 145).
} 
wendig im Modus der intra- und intermedialen Bezugnahme von Zeichen auf Zeichen, bzw. von Medien auf Medien vollziehen, so ist offensichtlich, dass in den Sprachspielen der kulturellen Semantik die Stadien der unproblematischen Geltung von Sinn von jenen unterschieden werden müssen, in denen das Spiel transkriptiver Bezugnahmen, der kulturellen Rekonzeptualisierungen, jeweils immer wieder in Gang kommt, wobei auch in die Stadien und Momente ,ungestörter' Semantik ,Störung' untilgbar als jene andauernde Fragilität eingeschrieben ist, von der jederzeit Prozesse der Remediation ihren Ausgang nehmen können. Ich möchte diese beiden Stadien medialer Kommunikation, das der unproblematischen Geltung von Sinn und das seiner jeweiligen Rekonzeptualisierung, - was ich an anderer Stelle ausführlicher diskutiert habe - als Stadien der ,Transparenz' und der ,Störung ${ }^{6}$ unterscheiden. ${ }^{38}$

Störung und Transparenz sollen dabei verstanden werden als zwei polare funktionale Zustände medialer Performanz, die konstitutiv eingeschrieben sind in das Verfahren der intra- und intermedialen Transkription. Transkription ließe sich dann beschreiben als der jeweilige Übergang von Störung zu Transparenz, von De- zu Rekontextualisierung der fokussierten Zeichen/Medien. Während Störung als Ausgangspunkt das transkriptive Verfahren der Remediation in Gang setzt und das Zeichen/Medium als (gestörter) Operator von Sinn in den Fokus der Aufmerksamkeit rückt, lässt sich Transparenz als der Zustand ungestörter medialer Performanz ansehen, in dem das jeweilige Zeichen/Medium mit Bezug auf den Gehalt, den es mediatisiert, verschwindet, transparent wird.

Transparenz soll also nicht - wie verschiedentlich in der medientheoretischen Debatte angenommen wurde - als eine quasi ontologische Eigenschaft von Medien angesehen werden, die - so etwa Engell und Vogl - dazu neigen, in Bezug auf das, was sie mediatisieren, ,gleichsam unwahrnehmbar, anästhetisch zu werden“, ${ }^{39}$ sondern vielmehr als ein funktionales Stadium symbolischer Interaktion, in dem die jeweils verhandelten Semantiken in ihrer Geltung unproblematisch sind und einen gleichsam ungestörten Realismus des Mediatisierten ermöglichen.

Realismus ist freilich, wie Goodman (1997, S. 45) feststellt, medienrelativ: „[E]r wird durch das Repräsentationssystem festgelegt, das für eine gegebene Kultur oder Person zu einer gegebenen Zeit die Norm ist", bzw. durch das mediale Dispositiv, in dem sich Kommunikation jeweils vollzieht. Der Realismus, mit dem symbolische Mittel repräsentieren, ist dabei umso höher, je vertrauter (transparenter) die gewählten Mittel sind. Mediale Darstellungen erscheinen uns dann realistisch, wenn „die Praxis [...] die Symbole so transparent [hat] werden lassen, daß wir uns einer Anstrengung

\footnotetext{
38 Vgl. Jäger (2004).

39 Vgl. Engell/Vogl (1999, S. 10); vgl. auch Krämer (2001, S. 157).
} 
oder irgendwelcher Alternativen oder der Tatsache, daß wir interpretieren, überhaupt nicht bewußt sind" (Goodman 1997, S. 44). In kommunikativen Zuständen dieser Art wird die Mediatisiertheit des Realen „durch unsere Neigung verschleiert, einen Bezugsrahmen dann nicht zu spezifizieren, wenn er unser eigener ist" (ebd., S. 45). Es zeigt sich hier, dass - wie Bolter/ Grusin (2001, S. 53) vermuten - in Mediensysteme grundsätzlich das Begehren eingeschrieben ist, „hinter die Grenzen der Repräsentation zu gelangen und das Reale selbst zu erreichen".

,Störung" soll also jeder Zustand im Verlauf einer Kommunikation heiBen, der bewirkt, dass ein Zeichen/Medium (operativ) seine Transparenz verliert und in seiner Materialität wahrgenommen wird, und ,Transparenz jeder Zustand, in dem die Kommunikation nicht, gestört' ist, also das Zeichen/Medium als Medium nicht im Fokus der Aufmerksamkeit steht.

Die These, die hier vertreten wird, ist also die, dass die Transparenz des Mediums keine ,Eigenschaft ${ }^{c}$ des Mediums ist, sondern ein Aggregatzustand, den das Medium dann annimmt, wenn die mediatisierte Semantik als stilles Wissen kommunikativ nicht, gestört' ist, ebenso wie umgekehrt Störung kein parasitärer Defekt der Kommunikation ist, sondern jener kommunikative Aggregatzustand, in dem das Zeichen/Medium als solches sichtbar und damit semantisierbar wird, jener Zustand also, der, wenn er eintritt, immer mit Remediatisierungs-, d.h. Transkriptionsbedarfen verknüpft ist, ohne dass dieser Prozess der ständigen Transformation fragiler semantischer Gleichgewichtszustände jemals unterbrochen werden könnte. Intra- und intermediale Transkriptivität markieren insofern im Bereich der kulturellen Semiosis ein Feld medialer Operationen, die fortwährend zwischen der fragilen Geltung unproblematischer Semantik auf der einen sowie Prozessen transkriptiver Rekonzeptualisierungen auf der anderen Seite oszillieren.

\section{Kleines Resümee}

Meine bisherigen Sondierungen auf dem Feld der epistemologischen und semiologischen Voraussetzungen einer Theorie der Intermedialität lassen sich in aller Vorläufigkeit so zusammenfassen, dass die Zeichen- und Mediensysteme, aus denen sich die Semantik von Kulturen speist, über keine systemtranszendenten Quellen für den Prozess der Konstitution und Evidenzauszeichnung von Sinn verfügen. Hierin liegt ihre Fragilität und Brüchigkeit und zugleich die Notwendigkeit, fortwährend in Verfahren der remediatisierenden Bezugnahme einzutreten. Die Verfahren der kulturellen Semiosis sind deshalb notwendigerweise darauf verwiesen, im Falle der Irritation, der Störung etablierter Semantiken, auf immer schon semiologisch konstituierten Sinn zurückzugreifen und ihn unter je spezifischen 
diskursiven Bedingungen „kulturell zu rekonzeptualisieren“, ${ }^{40}$ d.h. den Bedingungen der Verfahren transkriptiver Bezugnahme zu unterwerfen. Intraund intermediale Transkriptionen reagieren dabei etwa auf Resonanzstörungen, auf Verschiebungen in den Ökonomien individueller und kultureller Aufmerksamkeit, ${ }^{41}$ d.h. auf Irritationen in jenen ,übergeordnete[n] kulturelle[n] Optionen, Wertsetzungen, Institutionen", die - wie Aleida Assmann (2001, S. 17) gezeigt hat - „für die spezifische Ausformung von [kultureller] Aufmerksamkeit mit verantwortlich sind. ${ }^{\text {“42 }}$ Sie reagieren aber auch auf die Umbrüche, von denen die politischen und kulturellen Rahmenbedingungen von medialen Dispositiven immer wieder betroffen werden. Immer dann gerät das Spiel medialer Bezugnahmepraktiken in Bewegung: In den Diskursen der kulturellen Semiosis erodiert nun die unproblematische Geltung tradierter Semantiken, die in ihrer Evidenz ${ }^{43}$ irritiert und als irritierte in das mediale Spiel der transkriptiven Maschine eingespeist werden. Es ist also könnte man sagen - eine Epistemologie der Störung, die in den Remediationen und Rekonzeptualisierungen der kulturellen Semantik untilgbar am Werke ist und die intra- und intermedialen Bewegungen symbolisch-medialer Systeme in Gang hält.

\section{Literatur}

Aigner, Carl (1993): Diskurse der Bilder. Photokünstlerische Appropriationen kunsthistorischer Werke. In: Seipel (Hg.), S. 38-42.

Amelunxen, Hubertus von (1993): Aneignung und Simulakrum. Die Einholung des Anderen in der Fotografie. In: Seipel (Hg.), S. 23-37.

Assmann, Aleida (2001): Einleitung. In: Assmann/Assmann (Hg.), S. 11-24.

Assmann, Aleida/Assmann, Jan (Hg.) (2001): Aufmerksamkeiten. (= Archäologie der literarischen Kommunikation VII). München.

Assmann, Jan (1992): Das kulturelle Gedächtnis. Schrift, Erinnerung und politische Identität in frühen Hochkulturen. München.

Boehm, Gottfried (Hg.) (1995): Was ist ein Bild? (= Bild und Text I). 2. Aufl. München.

Boehm, Gottfried/Mersmann, Birgit/Spies, Christian (Hg.) (2008): Movens Bild. Zwischen Evidenz und Affekt. München.

Bolter, Jay David/Grusin, Richard (2001): Remediation. Understanding new media. 4. Aufl. London.

Brandom, Robert B. (2000): Expressive Vernunft. Begründen, Repräsentation und diskursive Festlegung. Frankfurt a.M.

40 Vgl. Manovich (2001, S. 47).

41 Vgl. hierzu Franck (1998); ebenso Assmann/Assmann (Hg.) (2001).

42 Die Irritation semantischer Evidenz von Sinn ist bereits auf der Mikroebene interpersonaler Kommunikation der Auslöser von transkriptiven Operationen. Vgl. hierzu Jäger (2004).

43 Zum Begriff der semantischen Evidenz vgl. Jäger (2008b). 
Brandom, Robert B. (2001): Begründen und Begreifen. Eine Einführung in den Inferentialismus. Frankfurt a.M.

Buschmeier, Gabriele/Konrad, Ulrich/Riethmüller, Albrecht (Hg.) (2008): Transkription und Fassung in der Musik des 20. Jahrhunderts. Beiträge des Kolloquiums in der Akademie der Wissenschaften und der Literatur, Mainz, vom 5. bis 6. März 2004. Stuttgart.

Buss, Mareike/Habscheid, Stephan/Jautz, Sabine/Liedtke, Frank/Schneider, Jan Georg (Hg.) (2009): Theatralität des sprachlichen Handelns. Eine Metaphorik zwischen Linguistik und Kulturwissenschaften. München.

Cassirer, Ernst (1964): Philosophie der symbolischen Formen. Darmstadt.

Danto, Arthur C. (1995): Abbildung und Beschreibung. In: Boehm (Hg.), S. 125-147.

Deacon, Terrence W. (1997): The symbolic species. The co-evolution of language and the brain. New York/London.

Derrida, Jacques (1974): Grammatologie. Frankfurt a.M.

Derrida, Jacques (1976): Die Schrift und die Differenz. Frankfurt a.M.

Derrida, Jacques (2003): Die Stimme und das Phänomen. Frankfurt a.M.

Engell, Lorenz/Vogl, Joseph (1999): Vorwort. In: Pias, Claus/Vogl, Joseph/Engell, Lorenz/Fahle, Oliver/Neitzel, Britta (Hg.): Kursbuch Medienkultur. Die maßgeblichen Theorien von Brecht bis Baudrillard. Stuttgart, S. 8-11.

Epping-Jäger, Cornelia/Linz, Erika (Hg.) (2003): Medien/Stimmen. (= Mediologie 9). Köln.

Fehrmann, Gisela/Jäger, Ludwig (2004): Sprachbewegung und Raumerinnerung. Zur topographischen Medialität der Gebärdensprache. In: Lechtermann, Christina/ Morsch, Carsten/Wenzel, Horst (Hg.): Kunst der Bewegung. Kinästhetische Wahrnehmung und Probehandeln in virtuellen Welten. (= Publikationen zur Zeitschrift für Germanistik 8). Bern, S. 311-341.

Fehrmann, Gisela/Linz, Erika/Schumacher, Eckhard/Weingart, Brigitte (2004): Originalkopie. Praktiken des Sekundären - Eine Einleitung. In: Fehrmann/Linz/Schumacher/Weingart (Hg.), S. 7-17.

Fehrmann, Gisela/Linz, Erika/Schumacher, Eckhard/Weingart, Brigitte (Hg.) (2004): Originalkopie. Praktiken des Sekundären. (= Mediologie 11). Köln.

Fehrmann, Gisela/Linz, Erika/Epping-Jäger, Cornelia (Hg.) (2005): Spuren - Lektüren. Praktiken des Symbolischen. München.

Franck, Georg (1998): Ökonomie der Aufmerksamkeit. München.

Fricke, Ellen (2008): Grundlagen einer multimodalen Grammatik des Deutschen: Syntaktische Strukturen und Funktionen. Habilitationsschrift, Europa-Universität Viadrina Frankfurt a.d. Oder. Ms.

Genette, Gérard (1993): Palimpseste. Die Literatur auf zweiter Stufe. Frankfurt a.M.

Goodman, Nelson (1997): Sprachen der Kunst. Entwurf einer Symboltheorie. Frankfurt a.M. 
Grésillion, Almuth (1995): Über die allmähliche Verfertigung von Texten beim Schreiben. In: Raible, Wolfgang (Hg.): Kulturelle Perspektiven auf Schrift und Schreibprozesse. Tübingen, S. 1-36.

Grube, Gernot/Kogge, Werner/Krämer, Sybille (Hg.) (2005): Schrift. Kulturtechnik zwischen Auge, Hand und Maschine. München.

Gülich, Elisabeth/Kotschi, Thomas (1996): Textherstellungsverfahren in mündlicher Kommunikation. Ein Beitrag am Beispiel des Französischen. In: Motsch, Wolfgang (Hg.): Ebenen der Textstruktur: Sprachliche und kommunikative Prinzipen. (= Reihe germanistische Linguistik 164). Tübingen, S. 37-80.

Gumbrecht, Hans Ulrich/Pfeiffer, K. Ludwig (Hg.) (1988): Materialität der Kommunikation. Frankfurt a.M.

Holly, Werner (2008): Audiovisuelle Sigetik. Über verborgene Bedeutungen im BildSprach-Zusammenhang. In: Pappert, Steffen/Schröter, Melani/Fix, Ulla (Hg.): Verschlüsseln, Verbergen, Verdecken in öffentlicher und institutioneller Kommunikation. Berlin, S. 147-169.

Humboldt, Wilhelm v. ([1936] 1968): Gesammelte Schriften, hg. v. d. Kgl. Preuß. Akad. d. Wiss. (Leitzmann, Gebhardt, Richter). 17 Bde. Berlin. [zitiert als Humboldt GS mit Band- und Seitenzahl].

Husserl, Edmund (1929): Formale und transzendentale Logik. Versuch einer Kritik der logischen Vernunft. Halle.

Jäger, Ludwig (1986): Der saussuresche Begriff des Aposème als Grundlagenbegriff einer hermeneutischen Semiologie. In: Jäger, Ludwig/Stetter, Christian (Hg.): Zeichen und Verstehen. Akten des Aachener Saussure-Kolloquiums 1983. (= Aachener Studien zur Semiotik und Kommunikationsforschung 3). Aachen, S. 7-33.

Jäger, Ludwig (1988): Über die Individualität von Rede und Verstehen. Aspekte einer hermeneutischen Semiologie bei Wilhelm von Humboldt. In: Frank, Manfred (Hg.): Individualität. (= Poetik und Hermeneutik XIII). München, S. 76-94.

Jäger, Ludwig (2000): Die Sprachvergessenheit der Medientheorie. Ein Plädoyer für das Medium Sprache. In: Kallmeyer (Hg.), S. 9-30.

Jäger, Ludwig (2001): Sprache als Medium. Über die Sprache als audio-visuelles Dispositiv des Medialen. In: Wenzel, Horst/Seipel, Wilfried/Wunberg, Gotthart (Hg.): Audiovisualität vor und nach Gutenberg - Zur Kulturgeschichte der medialen Umbrüche. (= Schriften des Kunsthistorischen Meseums 6). Wien, S. 19-42.

Jäger, Ludwig (2004): Störung und Transparenz. Skizze zur performativen Logik des Medialen. In: Krämer, Sybille (Hg.): Performativität und Medialität. München, S. 35-74.

Jäger, Ludwig (2005a): Vom Eigensinn des Mediums Sprache. In: Busse, Dietrich/Niehr, Thomas/Wengeler, Martin (Hg.): Brisante Semantik. Neuere Konzepte und Forschungsergebnisse einer kulturwissenschaftlichen Semantik. (= Reihe Germanistische Linguistik 259). Tübingen, S. 45-64.

Jäger, Ludwig (2005b): Versuch über den Ort der Schrift. Die Geburt der Schrift aus dem Geist der Rede. In: Grube/Kogge/Krämer (Hg.), S. 187-209. 
Jäger, Ludwig (2008a): Transkriptive Verhältnisse. Zur Logik intra- und intermedialer Bezugnahmen in ästhetischen Diskursen. In: Buschmeier/Konrad/Riethmüller (Hg.), S. 103-134.

Jäger, Ludwig (2008b): Indexikalität und Evidenz. Bemerkungen zum Problem der deiktisch-indexikalischen Bezugnahme. In: Wenzel, Horst/Jäger, Ludwig (Hg.) (in Zusammenarb. m. Robin Curtis u. Christina Lechtermann): Deixis und Evidenz. (= Rombach-Wissenschaften, Reihe Scenae 8). Freiburg i.Br., S. 289-315.

Jäger, Ludwig/Linz, Erika (Hg.) (2004): Medialität und Mentalität. Theoretische und empirische Studien zum Verhältnis von Sprache, Subjektivität und Kognition. München.

Kallmeyer, Werner (2000a): Vorwort. In: Kallmeyer (Hg.), S. VII-IX.

Kallmeyer, Werner (2000b): Sprache und neue Medien - zum Diskussionsstand und zu einigen Schlussfolgerungen. In: Kallmeyer (Hg.), S. 292-315.

Kallmeyer, Werner (Hg.) (2000): Sprache und neue Medien. Jahrbuch des Instituts für Deutsche Sprache 1999. Berlin/New York.

Kendon, Adam/Müller, Cornelia (Hg.) (2007 ff.): Gesture Studies. Amsterdam/Philadelphia. [Buchreihe].

Kolesch, Doris/Krämer, Sybille (Hg.) (2006): Stimme. Annäherung an ein Phänomen. Frankfurt a.M.

Krämer, Sybille (2001): Sprache, Sprechakt, Kommunikation. Frankfurt a.M.

Krämer, Sybille (2004): Performativität und Medialität. München.

Krämer, Sybille/Kogge, Werner/Grube, Gernot (Hg.) (2007): Spur. Spurenlesen als Orientierungstechnik und Wissenskunst. Frankfurt a.M.

Kristeva, Julia (1969): Sēmeiotikē. Recherches pour une sémanalyse. Paris.

Latour, Bruno (1996): On actor-network-theory. A few clarifications. In: Soziale Welt 47, S. 369-381.

Leopold, Silke (1992): Einleitung: In Leopold (Hg.), S. 7-10.

Leopold, Silke (Hg.) (1992): Musikalische Metamorphosen. Formen und Geschichte der Bearbeitung. (= Bärenreiter Studienbücher zur Musik 2). Kassel.

Luhmann, Niklas (1992): Die Wissenschaft der Gesellschaft. Frankfurt a.M.

Luhmann, Niklas (1997): Die Gesellschaft der Gesellschaft. Frankfurt a.M.

Manovich, Lev (2001): The language of new media. London.

Mitchell, William J.T. (1995): Picture theory. Chicago/London.

Mitchell, William J.T. (2008): Bildtheorie. Hrsg. u. m. e. Nachwort v. Gustav Frank. Frankfurt a.M.

Münker, Stefan/Roesler, Alexander (Hg.) (2008): Was ist ein Medium? Frankfurt a.M.

Pape, Helmut (1999): Indexikalität der Erfahrung oder Objektivität des Wissens. In: Zeitschrift für Semiotik 21/1, S. 3-14. 
Peirce, Charles S. (1970): Schriften II. Vom Pragmatismus zum Pragmatizismus, mit einer Einführung hrsg. v. Karl-Otto Apel. Frankfurt a.M.

Peirce, Charles S. (1984): Writings. A chronological edition. Vol. 2: 1867-1871. Bloomington.

Raible, Wolfgang (1995): Arten des Kommentierens - Arten der Sinnbildung - Arten des Verstehens. Spielarten der generischen Intertextualität. In: Assmann, Jan/Gladigow, Burkhard (Hg.): Text und Kommentar. Archäologie der Literarischen Kommunikation IV. München, S. 51-73.

Rorty, Richard (1994): Der Spiegel der Natur: Eine Kritik der Philosophie. 3. Aufl. Frankfurt a.M.

Saussure, Ferdinand de (1974): Cours de linguistique générale. Édition critique par Rudolf Engler. Tome 2, fascicule 4, Appendice. Wiesbaden.

Saussure, Ferdinand de (2003): Wissenschaft der Sprache. Neue Texte aus dem Nachlaß, herausgegeben und mit einer Einleitung versehen von Ludwig Jäger, übersetzt und textkritisch bearbeitet von Elisabeth Birk und Mareike Buss. Frankfurt a.M.

Seipel, Wilfried (Hg.) (1993): Diskurse der Bilder. Photokünstlerische Reprisen kunsthistorischer Werke. Katalog Kunsthistorisches Museum Wien. Wien.

Selting, Margret (1987): Reparaturen und lokale Verstehensprobleme oder: Zur Binnenstruktur von Reparatursequenzen. In: Linguistische Berichte 108, S. 128-149.

Stetter, Christian (1997): Schrift und Sprache. Frankfurt a.M.

Stoichita, Victor I. (1998): Das selbstbewußte Bild - Vom Ursprung der Metamalerei. München. [frz. (1993): L'instauration du tableau - Métapeinture à l'aube des temps modernes].

Vansina, Jan (1973): Oral Tradition. A Study in Historical Methodology. 2. Aufl. Harmondsworth.

Waldenfels, Bernhard (1999): Vielstimmigkeit der Rede. Frankfurt a.M.

Winkler, Hartmut (1997): Docuverse. Zur Medientheorie der Computer. München.

Winkler, Hartmut (2004): Übertragen - Post, Transport, Metapher. In: Fohrmann, Jürgen (Hg.): Rhetorik. Figuration und Performanz. Stuttgart/Weimar, S. 283-294.

Winkler, Hartmut (2008): Zeichenmaschinen. Oder warum die semiotische Dimension für eine Definition der Medien unerlässlich ist. In: Münker/Roesler (Hg.), S. 211-221.

Wirth, Uwe (2008): Die Frage nach dem Medium als Frage nach der Vermittlung. In: Münker/Roesler (Hg.), S. 222-234.

Wittgenstein, Ludwig (1984): Tractatus logico-philosophicus. In: Wittgenstein, Ludwig: Werkausgabe Bd. 1. Frankfurt a.M.

Zuschlag, Christoph (2002): Vom Kunstzitat zur Metakunst. Kunst über Kunst im 20. Jahrhundert. In: Mai, Ekkehard/Wettengl, Kurt (Hg.): Wettstreit der Künste. Malerei und Skulptur von Dürer bis Daumier. Köln, S. 171-189. 
